\title{
Exploring the Use of Google Glass in Wet Laboratories
}

\author{
Grace Hu \\ Wellesley College \\ 106 Central St \\ Wellesley, MA 02481 USA \\ ghu@wellesley.edu \\ Lily Chen \\ Wellesley College \\ 106 Central St \\ Wellesley, MA 02481 USA \\ Ichen8@wellesley.edu

\section{Johanna Okerlund} \\ Wellesley College \\ 106 Central St \\ Wellesley, MA 02481 USA \\ jokerlun@wellesley.edu \\ Orit Shaer \\ Wellesley College
106 Central St
Wellesley, MA 02481 USA
oshaer@wellesley.edu \\ Wellesley College
106 Central St
Wellesley, MA 02481 USA
oshaer@wellesley.edu \\ Wellesley College
106 Central St
Wellesley, MA 02481 USA
oshaer@wellesley.edu \\ Wellesley College
106 Central St
Wellesley, MA 02481 USA
oshaer@wellesley.edu
}

Permission to make digital or hard copies of part or all of this work for personal or classroom use is granted without fee provided that copies are not made or distributed for profit or commercial advantage and that copies bear this notice and the full citation on the first page. Copyrights for third-party components of this work must be honored. For all other uses, contact the Owner/Author.

Copyright is held by the owner/author(s)

CHI'15 Extended Abstracts, Apr 18-23, 2015, Seoul, Republic of Korea ACM 978-1-4503-3146-3/15/04.
Abstract

We present results from an exploratory field study of using Google Glass for training future scientists in wet laboratory work. Our goal is to investigate the potential of Glass for mitigating challenges of laboratory work and for increasing the confidence and efficiency of novice researchers. Our findings indicate how Glass is used in laboratory settings and highlight potential uses for Glass including hands-free interaction with experimental protocols and process documentation. We also discuss technical and usability limitations of using Glass in laboratory settings.

\section{Author Keywords}

Field study; qualitative, Google Glass, wet laboratory.

\section{ACM Classification Keywords}

H.5.m. Information interfaces and presentation (e.g., $\mathrm{HCI}$ ): Miscellaneous.

\section{Introduction}

Wet laboratory work is essential for training future scientists in various areas including Biology and Chemistry. Experience with lab work, which requires researchers to handle chemical and biological matter in wet states, provides students with opportunities to gain practical skills, apply knowledge in a hands-on environment, and receive personal mentorship [2]. Student researchers often learn by following protocols, 
checking off individual steps on paper, documenting observations in their lab notebooks, and interacting with peers and mentors in the lab [2].

Over the last three years our research group has established a design partnership with the Cidar Lab, a synthetic biology laboratory at Boston University, and with a biology summer research program at our institution. Our goal is to investigate the potential of emerging user interface technologies for mitigating challenges associated with laboratory work and for increasing the confidence and efficiency of novice researchers. Over three summers, we observed student researchers in wet laboratory settings.

In the lab, student researchers work with a wide variety of lab instruments, machines, and substances while documenting their work in lab notebooks. Researchers use timers to keep track of processes and follow paperbased experimental protocols closely, checking off steps as they progress. Lab activities occur simultaneously, across different areas in the lab so researchers move around frequently, often carrying substances and lab notebooks. We observed that novice researchers often struggle to keep track of the various space- and time- multiplexed activities. Space in small research labs is limited. The workbench is typically crowded as it is shared by 3-6 peers and is often cluttered with instruments and lab ware. In the lab, safety is a critical issue, and researchers must wear lab gear, such as gloves, goggles, and coats. These limitations often hinder the mobility and efficiency of novice researchers.

In this paper, we present results from an exploratory qualitative field study with 11 participants that investigated the use of Google Glass for training novice researchers in wet laboratory work. Glass [4] is a wearable technology that resembles off-the-shelf glasses. It includes an optical display above the right eye and Bluetooth connectivity. Glass supports multiple input modalities using a touchpad, camera, microphone, speakers, and sensors. Our focus is on investigating Glass' potential for mitigating challenges associated with wet laboratory work in bio-safety level 1 laboratories (lowest level of bio containment precaution required) and for increasing the confidence and efficiency of novice researchers.

\section{Related Work}

Several projects investigated augmented capture and situated access to biological data. Labscape [1] is a smart environment for cell biologists. Mackay et al. [5] and Tabard et al. [9] explored augmenting biologists' paper notebooks by combining physical and digital sources of information. Schraefel et al. [8] developed a tablet-based system for the chemistry lab that replaces paper and enables recording the execution of experiments. eLabBench [10] explored the feasibility of integrating tabletop interfaces as interactive wet lab benches. However, tabletop interfaces limit the interaction to an interactive surface. Augmented reality systems, such as Glass, allow for interactions in the environment at large [3]. Glass has been studied in various contexts including surgery [6] and assistive technology [7]. Our study draws upon the methodology applied by these studies and further explores the situated use of wearable augmented reality devices, specifically in a wet lab setting. 


\section{Exploratory Field Study}

We identified three goals for our study: G1) Learning about the use of Glass in wet laboratory settings. Specifically: how is Glass used when users' hands are not available for touching or holding? In what scenarios and tasks is Glass used? What actions are performed? How does Glass support novice researchers in their lab work? Can it increase their confidence? G2) Understanding the technical implications and limitations of using Glass in wet labs; and G3) Inspiring designers and users to think about new needs and possibilities that emerge from everyday use in laboratory settings.

\section{Methodology}

We began by conducting observations in seven different biology labs led by our design partners. Then we conducted design workshops with each of our partners: overall 2 primary investigators (PIs), 1 graduate student, and 14 undergraduate students participated in one of 5 workshops.

In the workshop, we showed participants a promotional video of Glass, followed by a demonstration of Glass. To evoke discussion, we demonstrated the following applications: camera, video, timer, stopwatch, and starting from the second workshop - an early prototype of wet-lab protocol application that we developed (Figure 1). Each participant was then given the opportunity to try Glass for a short period of time. We concluded the workshop with a moderated group discussion of potential uses for Glass in wet laboratory. The discussion was recorded analysis methods. First level codes were developed from preliminary review of the data by three independent coders and were then collapsed into advanced categories and themes based on frequency.
Several themes emerged from the workshop data, reflecting needs that could potentially be addressed with Glass: 1) Documenting lab work through notes, pictures, and video; 2) Viewing situated and timely information such as steps of an experimental protocol; 3) Managing and monitoring synchronous lab activities; 4) Supporting hands-free interaction while working with substances and contaminants; and 5) Complying with safety requirements such as wearing gloves and protective eyewear. These themes are also consistent with a concept video created by Digital Science ${ }^{1}$. Informed by these themes, we developed a prototype application for a field study.

In the first stage, we conducted a series of three-days long field trials in which three undergraduate researchers ( 1 female) used Glass while working in the lab. For the trials we provided participants with a Glass installed with timer, stopwatch, battery life notification, our prototype application, and Gallari (a photo application that responds to gesture input). A Google Nexus 4 phone was connected to Glass. We observed each user in the lab on the first day and asked users to answer questions and make notes of their experience at the end of each day. We also conducted a debrief with each user at the end of the study period. Informed by the issues uncovered in this preliminary study, we iterated on the design of our prototype application.

In the second phase of the study, we extended the trial period for our first three users so that each used the Glass for an additional week. We also conducted a series of three-days long field trials with an additional 8 undergraduate student researchers. We installed the

\footnotetext{
${ }^{1}$ Concept video Digital Science: http://youtu.be/orXws080DiQ
} 


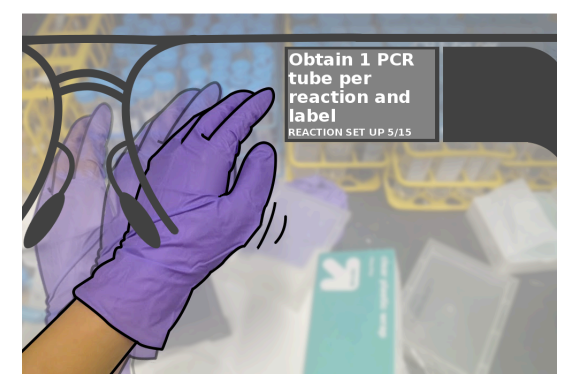

Figure 2. A user views a single protocol step and swipes their hand to navigate to the next one.

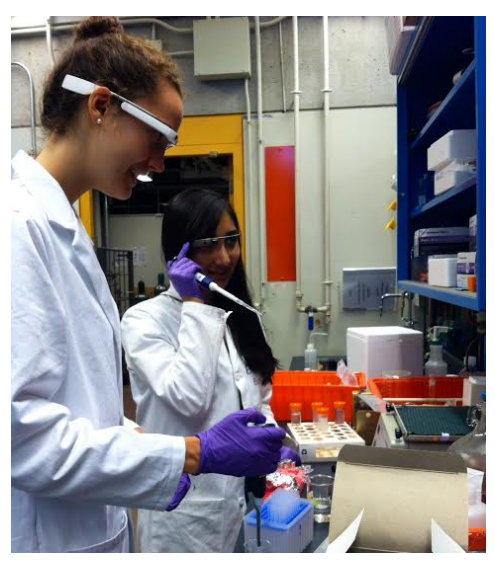

Figure 3. Student

researchers using Glass in wet laboratory. redesigned prototype application on the Glass in addition to the aforementioned timer and stopwatch. Participants were also encouraged to download other applications and to connect Glass to their contacts. We observed users in the lab on their first day and then asked them to answer questions about their experience at the end of each day. Finally, we debriefed with each user at the end of the study period.

\section{Prototype Application}

We developed a Google Glass application that allows users to navigate through experimental protocols using either hands-on or hands-free interaction techniques (Figure 2). Experimental protocols consist of a series of steps, each presented in a slide. For hands-on interaction, users swipe the built-in touch pad to navigate to an adjacent slide, tap to open a menu, and double tap to bookmark the current slide. For handsfree interaction, users use voice, head gestures, and hand gestures. To activate voice recognition, users nod up and speak "next," "previous," or "cancel" to change the slide. Users can also wave their hand in front of the camera from the right or left to navigate through the slides. The application is implemented using Android and Glass Development Kits.

In the initial version deployed for the first 3-day study, users could only navigate the protocols with touch commands and thus relied on the touchpad. Based on our initial findings, we improved the application to include voice recognition, head and hand gestures. Additional features we implemented in response to users' suggestions include viewing multiple steps at once and re-recalibration of hand and glove colors.

\section{Results}

Use Cases and Situated Actions

In the second phase of the study, 11 participants ( 9 female) used Glass for at least two hours a day.

Situated access to protocols - All participants used our developed application. Users viewed the following protocols: Miniprep (33 steps), Ligation (13 steps), and Transformation (20 steps). These protocols involve using various instruments across the lab. We observed that users often moved around the lab while carrying substances, leaving their notebooks and protocol sheets, relying solely on Glass for viewing instructions. Users used voice commands to proceed to the next step and occasionally to go back to or bookmark a step. Users indicated that the situated access to information supported their lab work, improved their mobility in the lab, and increased their confidence. In the words of one user: "I always find myself second-guessing myself in terms of what I'm adding to my samples and in what amount, but having the protocol in my line of sight streamlined my technique. I felt like I was able to do my procedure fluidly and confidently thanks to Glass."

Hands-Free Interaction - Working at the bench, users attempted to use gestures to navigate the application with partial success, which depended on lighting conditions and background. Users also tapped the glass, but found it inconvenient while wearing gloves.

Documenting Lab Work - Some participants used Glass for documenting their lab work using photos (4 users), video ( 2 users), and note taking with EverNote (2 users). These features were not integrated into the prototype app and required navigating to other Glass applications. Photos and videos were taken by tapping on Glass. While users indicated that touch-based interaction with Glass was inconvenient with gloves, they liked using Glass for documentation and expressed desire for a lab notebook feature in the prototype app. One user recounted: "I used the protocols you loaded 
for me. They looked beautiful on the Glass screen, and it was really neat to feel like I had my protocols at my fingertips. I also photographed some of the things that I was doing as a quasi-virtual lab notebook."

Managing synchronous lab activities - Users also used a Timer application to manage synchronous lab activities

First day avg Last day avg

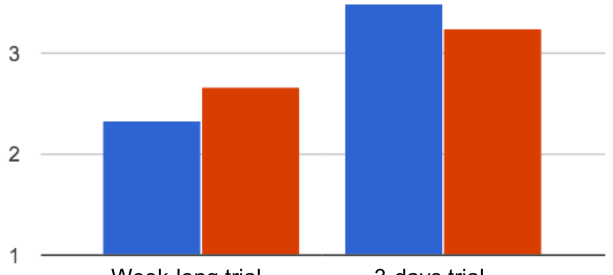

Week-long trial 3-days trial

Figure 4. Users' satisfaction of Glass at the beginning and end of the study on a Likert scale of five (1- Frustrated; would not recommend it, 5- Love it.) users) such as monitoring cell incubation while warming plates. This feature was also not part of the prototype app and required tapping on Glass to navigate to. We observed that users who did not use the Glass timer used physical timers in the lab. Users indicated a need for using Glass to monitor not only their own lab activities but also the activities of others so they can time their use of instruments.

In-office use - 7 participants also used Glass in their office space to check mail and share photos. Only one participant used it in social settings for directions.

Better with time?

Participants' satisfaction with Glass often increased after the first day of use: "I definitely liked Glass more the second day than the first, and I look forward to using it today." However, we also found evidence of novelty effect where interest and usage decreased after the first day. Figure 4 shows participants' average satisfaction ratings on the first and last days of the study. It is noticeable that, in general, satisfaction with Glass is only mild. We attribute this to the many usability issues discussed below.

\section{Usability Issues}

The use of Glass in situ highlighted several usability issues including: 1) Input Modality - Users reported that built-in voice commands worked well and the touch input was satisfactory but difficult to use when wearing gloves. Several users needed time to get used to the head-tilt gesture. The hand-gesture recognition suffered from several limitations including sensitivity to lighting, need for contrasting background, and occasional overheating of Glass; 2) Navigation - Glass' navigation structure requires users to swipe down on the touchpad to go back one level at a time until they reach the main menu. Thus users had to swipe numerous times to exit the application. This often posed a major annoyance and prevented some users from using features beyond the prototype app; 3) Battery Life - Users reported short battery life of 2-5 hours, which negatively affected the stability of our app. The battery often ran out without warning; and 4) Connectivity and Syncing - Users reported problems connecting Glass to the Internet when transitioning in and out of the lab. Others noted difficulties syncing their contacts and Google+ accounts.

\section{Compatibility with Lab Settings}

We evaluated Glass only within biosafety level 1 labs. In these settings, users are able to remove their gloves between experimental steps and are not required to constantly wear protective eyewear. We found users were able to successfully use Glass. One user used Glass with protective eyewear and reported relatively high satisfaction. Users expressed high satisfaction with voice commands and were able to use the touch input with and without gloves. However, PIs were not satisfied with touch input and indicated a strong need for hands-free interaction. It is clear that more robust gesture-based input implementation is required.

New needs and possibilities

The everyday use of the prototype app inspired users to think about new needs and possibilities. Beyond improved hand gesture recognition, users suggested the following: 1) Integrating protocol viewing, timer, and note taking into a single application; 2) Supporting 
user input so protocols can be updated on the fly; 3) Providing instructions for using the various instruments in the lab; 4) Facilitating the creation of a database of instructional videos recorded from the user's point of view, which are accessible based on context (activity and instruments involved); 5) Adding customized voice commands; 6) Providing a streamlined mechanism for uploading new protocols to Glass; and 7) Increasing team awareness by providing information about the status and use of various instruments upon gaze.

\section{Conclusions and Future Work}

This study explored the use of Glass for training future scientists in wet laboratories. Our findings shed light on how Glass can be used to help novice researchers in the lab by highlighting use cases and actions. Findings indicate potential for using Glass for situated access to information (e.g. protocols, instructions) and to document lab work in-process through hands-free interaction. Our study also points to technical and usability limitations that need to be addressed. While the scope of the study was small, it inspired users and designers to think about new needs and possibilities.

Future work will draw on the insights gathered here to develop a high-fidelity prototype of a laboratory Glass application, which supports protocol viewing, editing and process documentation. We plan to conduct a field study of 4 weeks with undergraduates participating in a summer research experience. We will make focused observations about whether and how the app provides a natural user experience. We would also like to study expert wet lab researchers to see if people more comfortable in the lab still find the new device helpful. Our goal is to explore the impact of using Glass on the efficiency, confidence, and learning of future scientists.

\section{Acknowledgements}

We thank Tracy Haddock and the BU iGEM team, and Wellesley College Science Center Research Program. This work was partially funded by NSF Grant No. IIS1149530 and by a grant from Agilent Technologies.

\section{REFERENCES}

1. Arnstein, L., C.-Y. Hung et al. 2002. Labscape: A Smart Environment for the Cell Biology Laboratory. IEEE Pervasive Computing 1, 3 (2002).

2. Baker, N., Verran, J. The future of microbiology laboratory classes - wet, dry or in combination? Nature Reviews Microbiology. 2(4) 2004.

3.Feiner, S.,, Macintyre, B., and Seligmann, D.. 1993. Knowledge-based augmented reality.Commun. ACM 36, 7 (July 1993), 53-62.

4. Google Glass. Retrieved Sept. 9, 2014 from http://www.google.com/glass/start/

5. Mackay, W., Letondal, C., Pothier, G., Bøegh K. and Sørensen, H. 2002. The missing link: augmenting biology laboratory notebooks. UIST 2002. ACM.

6. Muensterer, O., Lacher, M., Zoeller, C., et al. Google Glass in pediatric surgery: An exploratory study. Int J Surg 12(4). 2014

7. McNaney, R., Vines, J., Roggen, D. et al. 2014 Exploring the acceptability of Google Glass as an everyday assistive device for people with Parkinson's. In Proc. CHI 2014. ACM.

8. Schraefel, M., Hughes, G., Mills, H. et al. Breaking the book: translating the chemistry lab book into a pervasive computing lab environment. ACM CHI 'O4.

9. Tabard A., Mackay W., Eastmond E. From individual to collaborative: the evolution of prism, a hybrid laboratory notebook. In Proc. CSCW 2008. ACM.

10.Tabard, A., Hincapie-Ramos, J.D., Esbensen, M., and Bardram, J.E. 2011. The elabbench: an interactive tabletop system for the biology laboratory. ITS 2011. ACM (2011). 